\title{
Investigating unilateral pleural effusions: the role of cytology
}

\author{
David T. Arnold ${ }^{1}$, Duneesha De Fonseka ${ }^{1}$, Siobhan Perry², Anna Morley ${ }^{3}$, \\ John E. Harvey ${ }^{3}$, Andrew Medford ${ }^{3}$, Mary Brett ${ }^{4}$ and Nick A. Maskell ${ }^{1}$
}

Affiliations: ${ }^{1}$ Academic Respiratory Unit, Bristol Medical School, Translational Health Sciences, Bristol, UK. ${ }^{2}$ Bristol School of Anaesthesia, Bristol, UK. ${ }^{3}$ North Bristol Lung Centre, North Bristol NHS Trust, Bristol, UK. ${ }^{4}$ Dept of Cellular Pathology, North Bristol NHS Trust, Bristol, UK.

Correspondence: David T. Arnold, Academic Respiratory Unit, Bristol Medical School, Translational Health Sciences, Bristol, BS10 5NB, UK. E-mail: arnold.dtaAgmail.com

@ERSpublications

Largest prospective study investigating unilateral pleural effusions; the value of cytology depends on the primary site http://ow.ly/u7Fo30lOQPD

Cite this article as: Arnold DT, De Fonseka D, Perry S, et al. Investigating unilateral pleural effusions: the role of cytology. Eur Respir J 2018; 52: 1801254 [https://doi.org/10.1183/13993003.01254-2018].

ABSTRACT The vast majority of undiagnosed unilateral pleural effusions have fluid sent for cytological analysis. Despite widespread use, there is uncertainty about its sensitivity to diagnose malignant pleural effusions (MPEs). Our aim was to ascertain the utility of cytology using a large prospective cohort.

Consecutive patients presenting with an undiagnosed unilateral pleural effusion were recruited to this UK-based study. All had pleural fluid sent for cytological analysis. Cytological sensitivity was based on the final diagnosis at 12 months, confirmed by two consultants.

Over 8 years, 921 patients were recruited, of which 515 had a MPE. Overall sensitivity of fluid cytology to diagnose malignancy was $46 \%$ (95\% CI 42-58\%). There was variation in sensitivity depending on cancer primary, with mesothelioma (6\%) and haematological malignancies (40\%) being significantly lower than adenocarcinomas (79\%). MPEs secondary to ovarian cancer had high pick-up rates (95\%). In asbestos-exposed males with exudative effusions, the risk of MPE was 60\%, but cytological sensitivity was $11 \%$.

This is the largest prospective study of pleural fluid cytology and informs discussions with patients about the likely requirement for investigations following thoracentesis. In patients presenting with a clinical suspicion of mesothelioma, cytological sensitivity is low, so more definitive investigations could be performed sooner. 


\section{Introduction}

Pleural fluid analysis with cytological assessment is a fundamental part of the investigation of unilateral pleural effusions. In Europe and North America, one of the commonest causes is primary or secondary pleural malignancy [1]. Identifying malignancy from pleural fluid cytology alone can spare patients from more invasive investigations, reduces healthcare costs, is important for staging and allows earlier progression to treatment. However, it has several drawbacks, including an uncertain sensitivity and extends the time (routinely between 5 and 7 days) before further investigations are organised [2].

The estimates of sensitivity for detecting malignancy from pleural fluid cytology vary greatly within guidelines, ranging from $40 \%$ to $87 \%[1,3]$. The reason for this variation is due to retrospective study designs [4-7], selective study inclusion criteria [8,9] and a variation in cytopathological methods. Additionally, most studies of cytological yield cited in guidelines are $>20$ years old. There has been a significant advance in immunohistochemical methods since then.

Better knowledge of the discriminative ability of pleural fluid cytology would allow not only more informed consultations with patients, but better planning of further investigations. This study uses a large prospective cohort of patients with undiagnosed unilateral pleural effusions to assess cytological sensitivity depending on cancer type and patient factors. It aims to inform practice for respiratory physicians when diagnosing malignant pleural effusions.

\section{Methods}

\section{Patients}

Consecutive patients referred to a single-centre pleural service with an undiagnosed unilateral pleural effusion were recruited to this prospective observational study. All patients had a diagnostic thoracentesis as part of normal clinical care and consented to having their demographic data, blood and pleural fluid results stored. The study received ethical approval from the South West regional ethics committee (08/ H0102/11). All patients were followed-up to 12 months or death (whichever occurred first) and were assigned a final diagnosis as to the pathology or pathologies most likely to be the cause of their effusion. The final diagnosis was agreed by two independent consultant respiratory physicians based on all the available clinical, histological and radiological information. Any areas of contention were re-examined till consensus was reached.

\section{Serum and pleural fluid analysis}

All patients had routine pleural fluid analysis at baseline, including protein, glucose, lactate dehydrogenase $(\mathrm{LDH}), \mathrm{pH}$, microbiology culture and cytology. Light et al.'s [10] criteria were used to distinguish exudative from transudative effusions. Predominant pleural fluid cell types were defined based on British Thoracic Society guidelines [1]. A lymphocyte- or neutrophil-predominant effusion was defined as the presence of $>50 \%$ of that cell type in the absence of $\geqslant 10 \%$ eosinophils, in which case the effusion was deemed eosinophilic. Any effusion not meeting any of the above criteria was classed "nonspecific", i.e. both lymphocytes and neutrophils $<50 \%$, eosinophils $<10 \%$, with another cell type predominating (e.g. mesothelial, blood or atypical cells). In addition, routine baseline blood tests were performed. The serum neutrophil/lymphocyte ratio (a widely used indicator of poor prognosis for malignancy [11]) was calculated by dividing the serum neutrophils $\left(10^{9}\right.$ cells $\left.\cdot \mathrm{L}^{-1}\right)$ by serum lymphocytes.

\section{Pleural fluid cytology and immunohistochemistry}

As per guidelines, $40 \mathrm{~mL}$ of pleural fluid was sent for cytological analysis where possible [1]. It is standard practice in our centre that after preparing slides from the centrifuged deposit, a formalin-fixed paraffin embedded cell block is produced from each pleural fluid cytology sample. All samples were reviewed by a consultant cytopathologist. Depending on the degree of clinical suspicion of malignancy and/or initial cytological assessment, immunostaining was requested. The panel of immunohistochemical stains often included epithelial membrane antigen to distinguish between malignant cells and reactive mesothelial cells. Markers to distinguish between adenocarcinoma cells (AUA1 or, in later years, BerEP4) and mesothelial cells (CK5/6 and calretinin) were frequently used. In cases of adenocarcinoma, further immunostaining was undertaken to assess the most likely primary site. These markers included CK7, CK20, TTF1, oestrogen receptor, progesterone receptor and Ca125. Overlap of staining patterns sometimes occurred, with variation in the exact panels used between patients, but generally this panel of immunohistochemical stains provided useful information for diagnosis. Flow cytometry for lymphoma was sent based on a previously published algorithm [12]. A full breakdown of positive immunohistochemical markers in malignant effusions is shown in online supplementary appendix 1. Samples that were "nondiagnostic" for malignancy were those where a diagnosis of malignancy was not made based on the cytological specimen, with the patient requiring further investigations or interval radiological follow-up. In this instance, and 
when malignancy was the most likely diagnosis, it was usual practice to proceed to definitive biopsy (e.g. thoracoscopy or computed tomography (CT)-guided biopsy), instead of repeating thoracentesis.

\section{Diagnostic criteria}

Predefined criteria were used to reach a 12-month diagnosis. Malignant effusions were diagnosed in the presence of any of the following criteria. 1) Malignant pleural fluid cytology or biopsy; 2) histologically confirmed pulmonary/extrathoracic malignancy with radiographic evidence of metastasis to ipsilateral pleura on CT; 3) radiological changes meeting Leung's criteria that have progressed in keeping with malignancy on interval CT scan in the correct clinical context; or 4) autopsy confirming pleural malignancy. For full details of diagnostic criteria for nonmalignant pathologies see online supplementary appendix 2 .

\section{Statistical analysis}

Descriptive statistics were used to summarise patient characteristics and clinical data. Sensitivity estimates with $95 \%$ confidence intervals were used to investigate the ability of pleural fluid cytology to detect malignancy. When comparing cytological sensitivity between two groups the classic z-test was used with $\mathrm{p}<0.05$ used to define significance. Pleural fluid characteristics amongst the cohort were reported using descriptive statistics, with differences between cytology diagnostic and nondiagnostic effusions assessed using the independent samples t-test. Survival (from study entry) was censored at December 20, 2017.

\section{Results}

\section{Patient demographics}

Between December 2008 and December 2016, 921 consecutive patients presenting with an undiagnosed unilateral pleural effusion were recruited. All had a diagnostic thoracentesis for standard pleural fluid investigations, with $40 \mathrm{~mL}$ of fluid sent for cytological analysis in the majority (median $40 \mathrm{~mL}$, interquartile range (IQR) $35-40 \mathrm{~mL}$ ). The cohort had a mean \pm SD age of $70.2 \pm 13.8$ years and had a male predominance. The baseline characteristics of the cohort are shown in table 1.

\section{Effusion diagnoses}

The majority of effusions had a malignant aetiology at 12-month consultant diagnosis (56\%) (table 1). There were six patients in whom the exact cause of the effusion could not be ascertained. In all six, malignancy was excluded given resolution of effusion on follow-up imaging, so these cases have been placed in the nonmalignant group for further analysis. Table 2 shows the breakdown of the malignant effusions by primary site. Lung was the most numerous cancer primary causing effusions within this cohort $(32 \%, 166$ out of 515), with effusions secondary to mesothelioma accounting for $29 \%$ (148 out of 515) of the malignant diagnoses.

\section{Cytological sensitivity by cancer primary}

The sensitivity (95\% CI) of pleural fluid cytology for detecting different cancer types is shown in table 2 and figure 1. Cytology has a higher sensitivity for detecting adenocarcinomas compared to

\begin{tabular}{lccc} 
TABLE 1 Demographics & & & \\
& All & Malignant & Nonmalignant \\
\hline Subjects & 921 & 515 & 406 \\
Mean age years & 70 & 72 & 68 \\
Sex M:F & $601: 320$ & $317: 198$ & $284: 122$ \\
Laterality L:R & $385: 536$ & $222: 293$ & $163: 243$ \\
Previous malignancy & 212 & 176 & 36 \\
Survival days & $474(127-1632)$ & $199(74-465)$ & 1700 (831-2522) \\
Asbestos exposure & 274 & 166 & 108 \\
Pleural fluid analysis & $118(13)$ & $21(4)$ & $97(24)$ \\
$\quad$ Transudate & $315(34)$ & $183(35)$ & $132(33)$ \\
Predominant pleural fluid cell type & $87(9)$ & $13(3)$ & $74(18)$ \\
$\quad$ Lymphocytic & $71(8)$ & $30(6)$ & $41(10)$ \\
$\quad$ Neutrophilic & $448(49)$ & $289(56)$ & $159(39)$ \\
$\quad$ Eosinophilic & & & \\
$\quad$ Nonspecific & & & \\
\end{tabular}

Data are presented as $\mathrm{n}$, median (interquartile range) or $\mathrm{n}(\%)$. M: male; F: female; L: left; R: right. 
TABLE 2 Cytological sensitivity by cancer type

\begin{tabular}{lccc} 
& Cohort & Pleural fluid cytology diagnostic & Sensitivity \\
\hline All & 515 & 239 & $46.4(42.0-58.2)$ \\
Breast & 58 & 41 & $70.7(57.3-81.9)$ \\
ENT & 7 & 1 & $14.3(0.4-57.9)$ \\
Gastrointestinal & 22 & 15 & $68.2(45.1-86.1)$ \\
Haematological & 30 & 12 & $40.0(22.6-59.4)$ \\
Lung lall) & 166 & 93 & $56.0(48.1-63.7)$ \\
$\quad$ Adenocarcinoma & 100 & 82 & $82.0(73.1-89.0)$ \\
Squamous & 28 & 4 & $14.3(4.0-32.7)$ \\
Small cell & 16 & 7 & $43.8(19.8-70.1)$ \\
Other/unknown & 22 & 0 & $0(0-15.4)$ \\
Mesothelioma & 148 & 9 & $6.1(2.8-11.2)$ \\
Sarcoma/melanoma & 8 & 0 & $0(0-36.9)$ \\
Ovarian & 38 & 36 & $94.7(82.2-99.4)$ \\
Urological & 17 & 2 & $11.8(1.5-36.4)$ \\
Unknown malignancy & 21 & 7 & $33.3(14.6-57.0)$ \\
\hline
\end{tabular}

Data are presented as $\mathrm{n}$ or $\%(95 \% \mathrm{CI})$. ENT: ear, nose and throat.

other cancer types, even once mesothelioma is excluded $(\mathrm{p}<0.01)$. Within adenocarcinoma, there is a significant difference depending on cancer primary, with ovarian cancer having a significantly higher diagnostic rate than breast, lung or gastrointestinal malignancies, which all have similar sensitivities $(\mathrm{p}=0.013)$. Mesothelioma had a low sensitivity for detection on pleural fluid cytology alone with $94 \%$ of patients requiring a definitive biopsy before a diagnosis could be made. Of the 30 patients with a malignant effusion secondary to haematological malignancy (23 patients with lymphoma and seven with leukaemia), fewer than half had clear evidence of malignancy on pleural fluid cytology. Flow

FIGURE 1 Scatter plot of sensitivity of pleural fluid cytology by malignancy lerror bars represent 95\% confidence intervals).

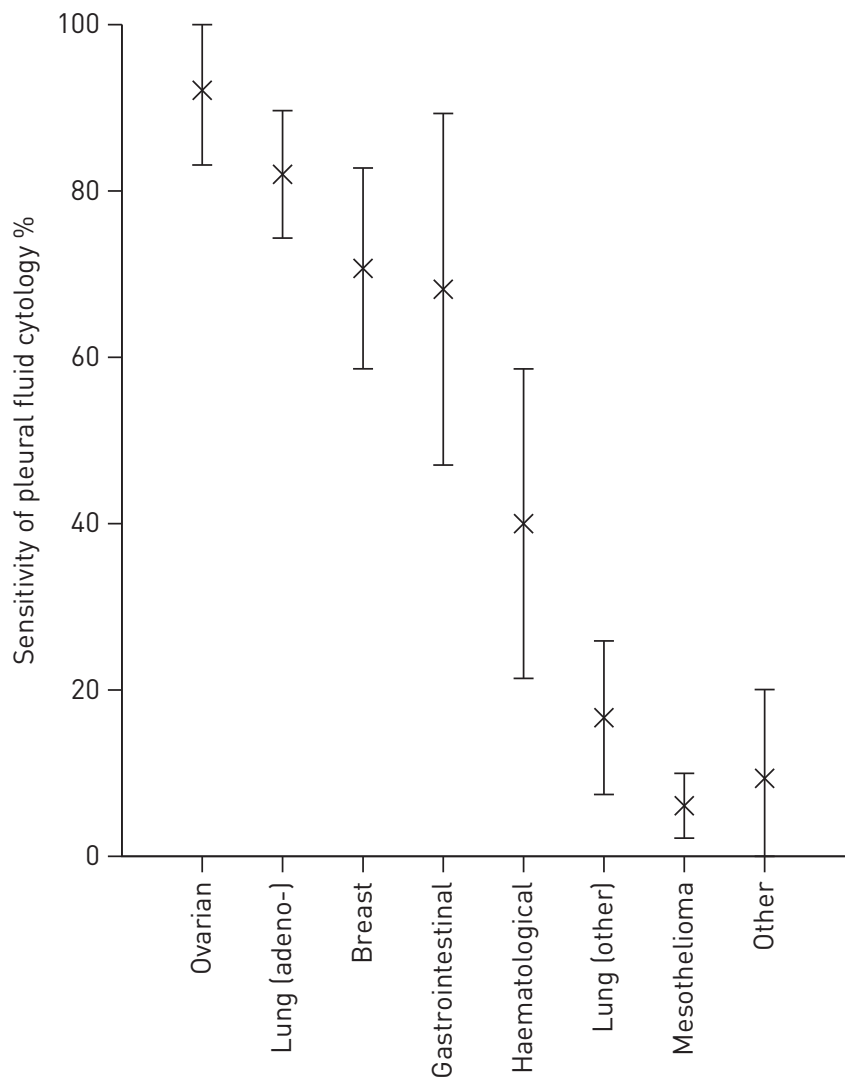

Primary malignancy 
cytometry was performed in 21 of these patients and assisted in the diagnosis of 16 . Malignant effusions from rarer primary sites such as urogenital, ear, nose and throat or musculoskeletal sites had low diagnostic rates, but numbers were small. Of the 276 nondiagnostic malignant pleural effusions, 248 (90\%) had a definitive histocytological diagnosis of malignancy (65\% pleural biopsy, $24 \%$ biopsy from nonpleural tumour site with radiographic evidence of metastatic pleural disease, $1 \%$ post-mortem). Pleural fluid cytology was repeated in 106 of these cases, often at the time of thoracoscopy or in patients unfit for more invasive investigations. Six (5.6\%) of these samples were diagnostic for malignancy. A third sample was sent for 30 patients, all of which were nondiagnostic. There was no difference in overall cytological sensitivity if more pleural fluid was sent for analysis. Overall sensitivity was $48 \%$ in samples of $\leqslant 40 \mathrm{~mL}$, compared to $40 \%$ in fluid samples $>40 \mathrm{~mL}$ ( $\mathrm{p}=0.65)$.

\section{Immunohistochemistry/cytogenetic results}

The full results of positive immunohistochemistry and cytogenetic markers are shown in online supplementary appendix 1 . It is of note that cytogenetic practice has advanced significantly during the course of the 8 years of recruitment. Therefore, certain tests, e.g. epidermal growth factor receptor, only became available towards the end of the study period, and were only requested if clinically indicated. There were 41 instances where further genetic information was request on the pleural fluid cell block. Two (5\%) of the cell blocks had insufficient material for further analysis.

\section{Diagnostic flow chart; risk of malignancy and sensitivity of pleural fluid cytology}

Figure 2 is a flowchart demonstrating the variation in the risk of malignancy and sensitivity of pleural fluid cytology depending on basic patient characteristics and pleural fluid analysis. These factors have been chosen as they are easily obtainable and have the greatest discriminative value in malignancy risk and/or cytological sensitivity. Whether an effusion is an exudate or transudate has a considerable bearing on the risk of malignancy. Within this cohort the risk of malignancy was 15\% (21 out of 118) in transudative effusions, compared to $62 \%$ (495 out of 803 ) in exudative effusions. Of the 21 patients with malignancy in the context of a transudative effusion, half $(n=11)$ had a concurrent diagnosis of cardiac failure. The malignancies were breast cancer $(n=2)$, lung cancer $(n=5)$, mesothelioma $(n=8)$, other types $(n=6)$.

The likelihood of malignancy in exudative effusions was $>60 \%$ and sensitivity of cytology remained $>40 \%$. Among female patients with an exudative effusion, the likelihood of malignancy was high (67\%), as was the sensitivity of cytology (66\%). Male patients with a previous history of cancer (excluding prostate cancer) had a high risk of malignancy and cytological sensitivity remained $>40 \%$.

Within the subgroup of asbestos-exposed male patients without a history of cancer, the sensitivity of pleural fluid cytology fell to $11 \%$ (95\% CI 6-17\%), which is significantly lower than other groups $(\mathrm{p}<0.01)$, despite a risk of malignancy of $>60 \%$. The patients with malignancy in this subgroup had a high likelihood of a "suspicion of malignancy" on their initial CT scan (117 out of 132).

\section{Survival: cytology diagnostic versus nondiagnostic malignant effusions}

The median (IQR) survival of all malignant effusions was 199 (74-465) days. There was considerable variation depending on cancer type, but there was no impact on survival between those with cytology diagnostic versus nondiagnostic effusions for individual cancers. For example, within lung adenocarcinoma, median (IQR) survival for cytology diagnostic effusions was 114 (47-281) days compared with 97 (IQR 32-201) days ( $\mathrm{p}=0.13$ ).

\section{Characteristics of cytology diagnostic versus nondiagnostic adenocarcinomas}

Cytology diagnostic malignant effusions secondary to adenocarcinoma were more likely to have serum or pleural markers of increased inflammation. This included a higher serum neutrophil/lymphocyte ratio, higher C-reactive protein, and a higher pleural fluid LDH. There was no significant difference in survival between the two groups $(\mathrm{p}=0.57)$ (table 3$)$.

\section{Discussion}

This is the largest ever prospective study examining the role of pleural fluid cytology in undiagnosed unilateral pleural effusions. With $>900$ patients, we can give an accurate assessment of the strengths and limitations of cytological assessment. The size of this cohort has allowed for analysis by cancer subtype and the construction of a diagnostic flowchart to demonstrate the likelihood of malignancy with the corresponding cytological sensitivity.

An unexplained pleural effusion is a common diagnostic challenge for the respiratory physician. In Europe and North America, a common cause is primary or secondary malignancy. Therefore, pleural fluid cytology is an essential aspect of pleural fluid analysis, but one that is poorly understood. It is recognised 


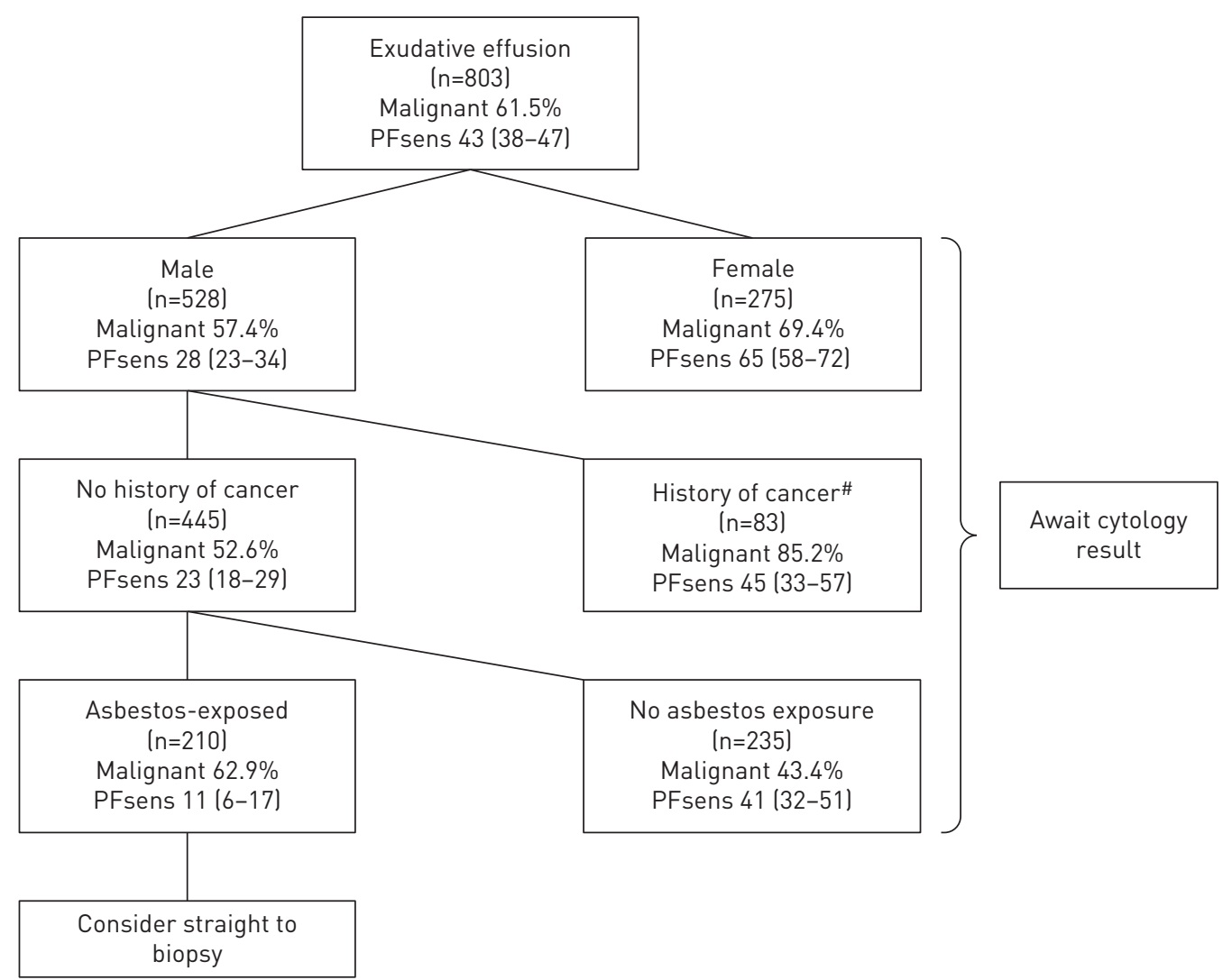

FIGURE 2 Diagnostic flow chart demonstrating risk of malignancy and sensitivity of pleural fluid cytology. Data are presented as \% (95\% CI), unless otherwise stated. PFsens: pleural fluid cytology sensitivity. \#: excluding prostate cancer.

that sensitivity is low, but estimates vary widely within international guidelines $(40-87 \%)[1,3]$. This variation arises because estimates are based on retrospective analyses of hospital or outpatient data [4-7]. PorCEL et al. [13] published a series of 3077 undiagnosed pleural effusions, of which 840 had a malignant aetiology. Overall, preliminary pleural fluid cytology was positive in $51 \%$ of malignant effusions, but due to geographical variation the prevalence of mesothelioma within the cohort was $<1 \%$, compared to $16 \%$ in our cohort. They also demonstrated that cytology was more accurate in adenocarcinoma of the lung (78\%), breast (68\%) and ovary (70\%). The data were collected retrospectively from 1994 to 2013, which could explain why estimates for sensitivity were lower than in the current study, given the advancement in immunohistochemical analysis. Retrospective series of lab cytology samples have been published, with very large numbers of subjects $(n>5000)$ [14-17]. These report the number of samples where malignant cells

\section{TABLE 3 Characteristics of cytology diagnostic versus nondiagnostic adenocarcinomas}

\begin{tabular}{lccc} 
& Diagnostic & Nondiagnostic & p-value \\
\hline Subjects & 173 & 45 & \\
Serum & & & \\
$\quad$ N/L ratio & $7.19 \pm 6.15$ & $5.05 \pm 2.41$ & $\mathbf{0 . 0 2}$ \\
C-reactive protein & $48.8 \pm 53.5$ & $30.4 \pm 27.8$ & $\mathbf{0 . 0 3}$ \\
Pleural fluid & & & \\
$\quad$ Protein & $44.9 \pm 9.2$ & $42.1 \pm 8.55$ & 0.08 \\
$\quad$ Glucose & $6.15 \pm 9.56$ & $5.86 \pm 1.66$ & 0.85 \\
LDH & $919.1 \pm 833.8$ & $644.9 \pm 706.9$ & $\mathbf{0 . 0 3}$ \\
pH & $7.38 \pm 0.17$ & $7.35 \pm 0.49$ & 0.70 \\
Survival & $148(56-425)$ & $98(40-241)$ & 0.574 \\
\end{tabular}

Data are presented as $n$, mean \pm SD or median (interquartile range), unless otherwise stated. Bold type represents statistical significance. N: neutrophil; L: lymphocyte; LDH: lactate dehydrogenase. 
were seen, which, although epidemiologically useful, is not linked to clinical information or final diagnosis so does not reflect a measure of sensitivity.

Two studies have prospectively recruited and followed-up patients to assess the accuracy of pleural fluid cytology. In 1979, HiRSCH et al. [18] recruited 300 patients who required diagnostic thoracentesis. All patients were routinely followed-up, but given the lack of modern diagnostics (i.e. CT scans) the final diagnosis was not identified in $20 \%$ of cases (compared to $0.6 \%$ in the current study). Malignancy was identified as a cause of the pleural effusion in 117 (39\%) patients. The sensitivity of pleural fluid cytology alone to identify malignancy was 54\% (95\% CI 44.4-63.1\%). Given the small numbers there was no subgroup analysis by cancer type or patient characteristics. A more recent study, from Thailand, prospectively recruited 353 patients who underwent a diagnostic thoracentesis [19]. There was a high prevalence of malignancy within the cohort (78\%) with one case of mesothelioma. Pleural fluid cytology was diagnostic in $61 \%$ (95\% CI 55.5-66.9\%) of cases, with a higher sensitivity in lung cancer $(73.7 \%)$ compared to nonlung solid cancers (53.5\%) and haematological malignancy (35.5\%). There was no further breakdown by cancer type or patient/fluid characteristics. However, the diagnostic criteria for malignancy were not robust with only $6 \%$ of cytology-negative malignant effusions having a definitive biopsy (compared to $90 \%$ in our cohort), with the remaining $94 \%$ being defined as cancerous following a "response to chemotherapy". This may account for the high prevalence of malignant effusions within this cohort and will significantly affect the estimate of sensitivity. Additionally, only $15 \mathrm{~mL}$ of pleural fluid was sent for cytological analysis, which is considerably less than recommended by guidelines $[1,20]$. In the current study, $40 \mathrm{~mL}$ of fluid was sent when possible. There was no significant difference in cytological sensitivity if less fluid was received, although numbers were small (44 samples $<40 \mathrm{~mL}$ ).

We have demonstrated that the overall sensitivity of pleural fluid cytology is slightly lower than the above prospective studies, at $45 \%$. The most likely reason for this is the high proportion of mesothelioma diagnoses in our cohort (29\%). This has a significant impact given the significant variability in sensitivity depending on cancer type. Mesothelioma was particularly low, with only $6 \%$ of cases being diagnosed on cytology alone. If the prevalence of mesothelioma is artificially lowered to be more in keeping with a typical European centre $(\sim 10 \%$ of all malignant effusions) the overall sensitivity of pleural fluid cytology rises to $55 \%$.

Some centres from areas with very high mesothelioma incidence report higher predictive values from pleural fluid cytology, but these are not commonplace [21, 22]. In most UK and European centres patients will require definitive biopsy unless there is clear evidence of malignant mesothelial cells with corroborative immunohistochemical markers, especially given the medicolegal implications of the diagnosis.

Cytological sensitivity from other cancers varied considerably by primary site and cell type. Adenocarcinomas from the breast, lung, ovary or gastrointestinal tract could be reliably detected on pleural fluid cytology alone (with a combined sensitivity of $80 \%$ ). Sensitivity approached $95 \%$ in ovarian cancer, which was significantly higher than other adenocarcinomas $(p=0.013)$. The pleura is the most common site for extra-abdominal spread in ovarian cancer [23]. It is hypothesised that most malignant effusions from ovarian cancer result from direct pleural invasion of the diaphragm, or the migration of malignant ascitic fluid through diaphragmatic defects [24]. This mode of spread may result in more malignant cells being present in fluid, as opposed to the other malignancies which cause effusions due to disrupting normal pleural fluid recycling at the parietal membrane [25].

We have investigated the variation in cytological sensitivity within adenocarcinomas alone and found that cytology diagnostic effusions correlate with biochemical markers indicative of more advanced/ inflammatory malignancy (higher serum neutrophil/lymphocyte ratio and C-reactive protein and pleural fluid LDH). Several previous smaller studies have correlated an increased cytological yield for other proxies of advanced tumours including lower pleural $\mathrm{pH}$ and glucose, macroscopic spread and survival [26-29]. It follows that more advanced tumours are likely to be cytology-positive due to increased exfoliation of tumour cells into the effusion. However, we did not find the same relationship between survival and cytology positivity when assessing individual tumour types. This finding from previous studies is likely to be because adenocarcinomas with higher cytological sensitivity have slightly better overall survival, e.g. breast and ovarian cancers [30].

This variation in the utility of pleural fluid cytology has significant implications for planning further investigations. Guidelines recommend waiting for the pleural fluid cytology result before proceeding to other invasive and costly investigations (e.g. local-anaesthetic thoracoscopy or CT-guided biopsy) [1]. This can take between 5 and 7 days (or longer if additional immunohistochemistry is required), and the patient may still be symptomatic without definitive pleural drainage. This study has shown that in asbestos-exposed male patients with no history of cancer, the likelihood of a diagnosing malignancy from an exudative effusion is just $6 \%$, despite the risk of malignancy being $>60 \%$. For this patient demographic 
we would support the approach of not waiting for the cytology result before performing a definitive biopsy. This is further supported by the finding that nearly $90 \%$ (117 out of 132) of the patients with a malignant effusion in this group had evidence of malignancy on their CT scan. In contrast, for patients not fulfilling these criteria, the higher sensitivity of pleural fluid cytology $(>40 \%)$ justifies waiting for the result.

This study has weaknesses that may limit the generalisability of its findings. However, although this was a single-centre study, the cytological and immunohistochemical techniques employed are in use in most European centres. Secondly, the cytopathologists were not blinded to the clinical information; they had information from the requesting clinician as well as from the multidisciplinary meeting. This may have influenced their interpretation of the cytology specimen, but this study is a pragmatic assessment of the value of pleural fluid cytology in day-to-day practice. Additionally, a concern when using pleural fluid cytology alone to diagnose malignancy is that there is insufficient material for further analysis. This is increasingly relevant given the continued development of targeted immunotherapy for malignancies that metastasise to the pleura. In our study, given the change in immunohistochemistry and cytogenetic practice over the 8-year recruitment period, the suitability of pleural fluid specimens for further analysis is difficult to quantify. In the 41 incidences where receptor status or genetic analysis was requested, the pleural fluid specimen was sufficient in 95\% (39 out of 41) of cases. Other studies with a focus on this issue have found that pleural fluid samples can reliably provide genetic information that correlates with the primary malignancy [31-34].

In conclusion, this is the largest prospective study of pleural fluid cytology in the literature. We have shown considerable variation in the sensitivity of cytological assessment by primary cancer type with adenocarcinoma, especially ovarian, having especially high sensitivity. Haematological malignancy and mesothelioma were unlikely to be diagnosed with pleural cytology alone. This information can help to inform discussions with patients around the likelihood of needing further investigations for pleural effusions. In asbestos-exposed male patients with an exudative effusion and no history of cancer, a strategy of not waiting for the cytology result before organising further tests is justifiable and would speed up the diagnostic and treatment pathway.

Acknowledgements: The authors would like to thank the patients, researchers and physicians who contributed to the pleural investigation study, on which this research was based.

Conflict of interest: None declared.

\section{References}

1 Hooper C, Lee YC, Maskell N, et al. Investigation of a unilateral pleural effusion in adults: British Thoracic Society Pleural Disease Guideline 2010. Thorax 2010; 65: Suppl. 2, ii4-i17.

2 Royal College of Pathologists. Key Performance Indicators in Pathology. Recommendations from the Royal College of Pathologists. https://rcpath.org/profession/clinical-effectiveness/key-performance-indicators-kpi.html Date last updated: April 2013.

3 Rivera MP, Mehta AC, Wahidi MM. Establishing the diagnosis of lung cancer: diagnosis and management of lung cancer, 3rd ed: American College of Chest Physicians evidence-based clinical practice guidelines. Chest 2013; 143: Suppl. 5, e142S-e165S.

4 Bielsa S, Panadés MJ, Egido R, et al. Rentabilidad del estudio citológico del líquido pleural en el derrame maligno. [Accuracy of pleural fluid cytology in malignant effusions]. An Med Interna 2008; 25: 173-177.

5 Nance KV, Shermer RW, Askin FB. Diagnostic efficacy of pleural biopsy as compared with that of pleural fluid examination. Mod Pathol 1991; 4: 320-324.

6 Prakash UB, Reiman HM. Comparison of needle biopsy with cytologic analysis for the evaluation of pleural effusion: analysis of 414 cases. Mayo Clin Proc 1985; 60: 158-164.

7 Salyer WR, Eggleston JC, Erozan YS. Efficacy of pleural needle biopsy and pleural fluid cytopathology in the diagnosis of malignant neoplasm involving the pleura. Chest 1975; 67: 536-539.

8 Renshaw AA, Dean BR, Antman KH, et al. The role of cytologic evaluation of pleural fluid in the diagnosis of malignant mesothelioma. Chest 1997; 111: 106-109.

9 Rodríguez-Panadero F. Medical thoracoscopy. Respiration 2008; 76: 363-372.

10 Light RW, Macgregor MI, Luchsinger PC, et al. Pleural effusions: the diagnostic separation of transudates and exudates. Ann Intern Med 1972; 77: 507-513.

11 Templeton AJ, McNamara MG, Šeruga B, et al. Prognostic role of neutrophil-to-lymphocyte ratio in solid tumors: a systematic review and meta-analysis. J Natl Cancer Inst 2014; 106: dju124.

12 Dixon G, Bhatnagar R, Zahan-Evans N, et al. A prospective study to evaluate a diagnostic algorithm for the use of fluid lymphocyte subset analysis in undiagnosed unilateral pleural effusions. Respiration 2018; 95: 98-105.

13 Porcel JM, Esquerda A, Vives M, et al. Etiology of pleural effusions: analysis of more than 3,000 consecutive thoracenteses. Arch Bronconeumol 2014; 50: 161-165.

14 Garcia LW, Ducatman BS, Wang HH. The value of multiple fluid specimens in the cytological diagnosis of malignancy. Mod Pathol 1994; 7: 665-668.

15 Gupta S, Sodhani P, Jain S. Cytomorphological profile of neoplastic effusions: an audit of 10 years with emphasis on uncommonly encountered malignancies. J Cancer Res Ther 2012; 8: 602-609.

16 Johnston WW. The malignant pleural effusion. A review of cytopathologic diagnoses of 584 specimens from 472 consecutive patients. Cancer 1985; 56: 905-909. 
17 Hsu C. Cytologic detection of malignancy in pleural effusion: a review of 5,255 samples from 3,811 patients. Diagn Cytopathol 1987; 3: 8-12.

18 Hirsch A, Ruffie P, Nebut M, et al. Pleural effusion: laboratory tests in 300 cases. Thorax 1979; 34: 106-112.

19 Assawasaksakul T, Boonsarngsuk V, Incharoen P. A comparative study of conventional cytology and cell block method in the diagnosis of pleural effusion. J Thorac Dis 2017; 9: 3161-3167.

20 Abouzgheib W, Bartter T, Dagher H, et al. A prospective study of the volume of pleural fluid required for accurate diagnosis of malignant pleural effusion. Chest 2009; 135: 999-1001.

21 Whitaker D. The cytology of malignant mesothelioma. Cytopathology 2000; 11: 139-151.

22 Segal A, Sterrett GF, Frost FA, et al. A diagnosis of malignant pleural mesothelioma can be made by effusion cytology: results of a 20 year audit. Pathology 2013; 45: 44-48.

23 Wimberger $\mathrm{P}$, Wehling M, Lehmann $\mathrm{N}$, et al. Influence of residual tumor on outcome in ovarian cancer patients with FIGO stage IV disease: an exploratory analysis of the AGO-OVAR (Arbeitsgemeinschaft Gynaekologische Onkologie Ovarian Cancer Study Group). Ann Surg Oncol 2010; 17: 1642-1648.

24 Porcel JM, Diaz JP, Chi DS. Clinical implications of pleural effusions in ovarian cancer. Respirology 2012; 17: 1060-1067.

25 Psallidas I, Kalomenidis I, Porcel JM, et al. Malignant pleural effusion: from bench to bedside. Eur Respir Rev 2016; 25: 189-198.

26 Pinelli V, Laroumagne S, Sakr L, et al. Pleural fluid cytological yield and visceral pleural invasion in patients with epithelioid malignant pleural mesothelioma. J Thorac Oncol 2012; 7: 595-598.

27 Rodríguez-Panadero F, López Mejías J. Low glucose and pH levels in malignant pleural effusions. Diagnostic significance and prognostic value in respect to pleurodesis. Am Rev Respir Dis 1989; 139: 663-667.

28 Rodriguez-Panadero F, Lopez-Mejias J. Survival time of patients with pleural metastatic carcinoma predicted by glucose and pH studies. Chest 1989; 95: 320-324.

29 Sahn SA, Good JT Jr. Pleural fluid $\mathrm{pH}$ in malignant effusions. Diagnostic, prognostic, and therapeutic implications. Ann Intern Med 1988; 108: 345-349.

30 Clive AO, Kahan BC, Hooper CE, et al. Predicting survival in malignant pleural effusion: development and validation of the LENT prognostic score. Thorax 2014; 69: 1098-1104.

31 Liu N, Sun RZ, Du J, et al. Comparison of epidermal growth factor receptor gene mutations identified using pleural effusion and primary tumor tissue samples in non-small cell lung cancer. Appl Immunohistochem Mol Morphol 2017; 26: e44-e51.

32 Kawahara A, Fukumitsu C, Azuma K, et al. A combined test using both cell sediment and supernatant cell-free DNA in pleural effusion shows increased sensitivity in detecting activating EGFR mutation in lung cancer patients. Cytopathology 2018; 29: 150-155.

33 Francis IM, Alath P, George SS, et al. Metastatic breast carcinoma in pleural fluid: correlation of receptor and HER2 status with the primary carcinoma - a pilot study. Diagn Cytopathol 2016; 44: 980-986.

34 Shabaik A, Lin G, Peterson M, et al. Reliability of Her2/neu, estrogen receptor, and progesterone receptor testing by immunohistochemistry on cell block of FNA and serous effusions from patients with primary and metastatic breast carcinoma. Diagn Cytopathol 2011; 39: 328-332. 